\title{
Comparison of Continuous Glucose Monitoring Accuracy Between Abdominal and Upper Arm Insertion Sites
}

\author{
Isabelle Isa Kristin Steineck, MD, ${ }^{1,2,4, *}$ Zeinab Mahmoudi, MS, PhD, 2,3,* \\ Ajenthen Ranjan, MD, PhD, 1,2,4,5 Signe Schmidt, MD, PhD, $1,2,4$ \\ John Bagterp Jørgensen, MS, PhD, ${ }^{3}$ and Kirsten Nørgaard, MD, DMSc ${ }^{1,4}$
}

\begin{abstract}
Background: The aim was to compare the accuracy of the Dexcom ${ }^{\circledR}$ G4 Platinum continuous glucose monitor (CGM) sensor inserted on the upper arm and the abdomen in adults.

Methods: Fourteen adults with type 1 diabetes wore two CGMs, one placed on the upper arm and one placed on the abdomen. Three in-clinic visits of $5 \mathrm{~h}$ with YSI (2300 STAT, Yellow Springs Instrument) measurements as comparator were performed. Each visit was followed by 4 days with seven-point self-monitoring of blood glucose (SMBG) in free-living conditions. Accuracy analyses on the paired CGM-YSI and CGM-SMBG measurements of the two CGM sensors were performed.

Results: Using YSI as comparator, the overall Mean Absolute Relative Difference (MARD) for the CGM abd $_{1}$ was $12.3 \%$ and $\mathrm{CGM}_{\text {arm }}$ was $12.0 \%$. The percentage of the CGM measurements in zone A of Clarke error grid analysis for the $\mathrm{CGM}_{\mathrm{abd}}$ was $85.6 \%$ and $\mathrm{CGM}_{\mathrm{arm}}$ was $86.0 \%$. The hypoglycemia sensitivity for the $\mathrm{CGM}_{\mathrm{abd}}$ and $\mathrm{CGM}_{\text {arm }}$ was $69.3 \%$. Using SMBG as comparator, the overall MARD for the $\mathrm{CGM}_{\text {abd }}$ was $12.5 \%$ and $\mathrm{CGM}_{\text {arm }}$ was $12.0 \%$. The percentage of the CGM measurements in zone A for the $\mathrm{CGM}_{\mathrm{abd}}$ was $84.1 \%$ and the $\mathrm{CGM}_{\text {arm }}$ was $85.0 \%$. The hypoglycemia sensitivity for the $\mathrm{CGM}_{\mathrm{abd}}$ was $60.0 \%$ and the $\mathrm{CGM}_{\text {arm }}$ was $71.1 \%$. All the $P$ values from the comparisons between the accuracy of $\mathrm{CGM}_{\mathrm{abd}}$ and $\mathrm{CGM}_{\mathrm{arm}}$ were $>0.05$.

Conclusion: The accuracy of a Dexcom G4 Platinum CGM sensor placed on the upper arm was not different from the accuracy of the sensor placed on the abdomen in adults with type 1 diabetes.
\end{abstract}

Keywords: Continuous glucose monitoring, CGM, Accuracy, Insertion sites, Abdomen, Arm.

\section{Introduction}

C ONTINUOUS GLUCOSE MONITORS (CGMs) have been available for almost 20 years. ${ }^{1}$ CGM tracks the glucose concentrations in the body's interstitial fluid and thereby provides near real-time glucose data. Near-normal glucose values reduce the risk of diabetes late complications and CGM facilitates the time spent in the target glucose range. ${ }^{2}$ CGM can warn users if glucose is trending toward hypo- or hyperglycemia, ${ }^{3}$ however, the accuracy of a CGM can be affected by rapid glucose changes, hyperglycemia, and hypoglycemia. Currently, the sensor for the Dexcom ${ }^{\circledR}$ G4 Platinum CGM is only approved for use on the abdomen for adults and the buttocks and abdomen for children. For newer versions of Dexcom the same applies. We have observed that several individuals place their CGMs on their upper arm. Until now, only one study reported the accuracy of placement of CGM on the upper arm; a study in very young children

\footnotetext{
${ }^{1}$ Department of Endocrinology, Copenhagen University Hospital Hvidovre, Hvidovre, Denmark.

${ }^{2}$ Danish Diabetes Academy, Odense, Denmark.

${ }^{3}$ Department of Applied Mathematics and Computer Science, Technical University of Denmark, Lyngby, Denmark.

${ }^{4}$ Steno Diabetes Center Copenhagen, Gentofte, Denmark.

${ }^{5}$ Department of Pediatrics, Herlev University Hospital, Herlev, Denmark.

*These two authors contributed equally to this work and are considered co-first authors.
} 
TABle 1. Baseline Characteristics FOR THE PARTICIPANTS

\begin{tabular}{lc}
\hline Variable, units & Median (range) \\
\hline Gender, females & 6 \\
BMI, kg/m 2 & $26(21-30)$ \\
Type 1 diabetes duration, years & $25(8-51)$ \\
Insulin pump duration, years & $7(1-35)$ \\
Age, years & $48(23-66)$ \\
HbAlc, mmol/mol & $53(43-65)$ \\
\hline
\end{tabular}

BMI, body mass index.

using self-monitoring of blood glucose (SMBG) as the comparator. $^{4}$

The aim of this study was to compare the accuracy of two Dexcom G4 Platinum CGMs in adults placed on either the abdomen or on the upper arm using two different reference methods, 2300 STAT, Yellow Springs Instrument (YSI) and SMBG. Our hypothesis was that the accuracy of the two Dexcom G4 Platinum CGMs placed at different sites would be identical.

\section{Materials and Methods}

The study was conducted at the research unit at the Department of Endocrinology, Copenhagen University Hospital Hvidovre, Hvidovre, Denmark. Fourteen adults went through a screening day, a run-in period and three in-clinic visits with 4 days of follow-up in free-living conditions. Study participants gave informed consent before inclusion. The study was monitored by the Good Clinical Practice unit at the Copenhagen University Hospital Bispebjerg, Denmark and approved by the Danish Health and Medicines Authority (EudraCT 2016-002127-28 and Medical Ref. No. 2016044243), the Regional Committee on Health Research Ethics (Protocol Code No. 16016762), and the Danish Data Protection Agency (2012-58-0004), was conducted in accordance with the Declaration of Helsinki, and was registered at clinicaltrials.gov (NCT02882737).

\section{Recruitment}

Participants were recruited from the outpatient diabetes clinic at Copenhagen University Hospital Hvidovre in the period September 2016 to May 2017. The inclusion criteria were as follows: age 18-70 years; body mass index 20$30 \mathrm{~kg} / \mathrm{m}^{2} ; \mathrm{HbA} 1 \mathrm{c} \leq 8.5 \%$ ( $\left.\leq 69 \mathrm{mmol} / \mathrm{mol}\right)$; duration of type 1 diabetes $\geq 2$ years; insulin pump treatment $\geq 1$ year, selfreported hypoglycemia awareness, and use of carbohydrate counting and the insulin pump bolus calculator for all meals. Participants were excluded if unable to stop paracetamol usage during the study period, pregnant or breast-feeding, performing vigorous intense aerobic exercise more than $3 \mathrm{~h}$ per week due to a second study performed simultaneously, ${ }^{5}$ or using medication besides insulin, influencing glucose metabolism.

\section{Screening}

The screening was performed in the morning after an overnight fast. Information was collected on sex, age, race, diabetes duration, allergies, medical history, and medications; height, weight, blood pressure, and heart rate were measured. The duration of insulin pump use, insulin pump settings, and total daily insulin dose were recorded. All participants used their own insulin pumps and did not change their pump settings between in-clinic visits.

\section{Insertions of two CGMs}

Around $48 \mathrm{~h}$ before each in-clinic visit, a CGM (Dexcom G4; Platinum, San Diego) was placed on the abdomen at least $7 \mathrm{~cm}$ from the site of the insulin pump infusion set. Our Dexcom G4 monitors were upgraded to the 505 software that is used in Dexcom G5 monitors. One CGM was placed on the nondominant upper arm between $10 \mathrm{~cm}$ from elbow and $10 \mathrm{~cm}$ from the shoulder on the posterior/lateral side on the arm. The CGM was calibrated by the participant at least every $12 \mathrm{~h}$ by SMBGs. The participant was told to do the calibration during a period of stabile blood glucose levels.

Participants were instructed to change their infusion sets the day before each in-clinic visit.

Table 2. Mean Absolute Relative Difference and Median Absolute Relative Difference for Euglycemia, Hyperglycemia, and the Whole Range

\begin{tabular}{|c|c|c|c|c|c|}
\hline Insertion site & Reference & Metric & Euglycemia & Hyperglycemia & Whole range \\
\hline Abdomen & YSI & MARD & $11.3(10.0)$ & $9.6(7.0)$ & $12.3(12.2)$ \\
\hline Arm & YSI & MARD & 12.4 (11.9) & $6.3(5.3)$ & $12.0(13)$ \\
\hline Abdomen & YSI & MedARD & $9.1[4.2-15.1]$ & $8.1[4.5-13.5]$ & $9.3[4.5-16.0]$ \\
\hline Arm & YSI & MedARD & $9.2[3.8-16.5]$ & $4.9[2.5-9.7]$ & $8.4[3.5-15.7]$ \\
\hline Abdomen & SMBG & MARD & $11.4(10.8)$ & $10.3(9.1)$ & $12.5(18.2)$ \\
\hline Arm & SMBG & MARD & $11.2(10.9)$ & $9.1(9.6)$ & $12.0(19.4)$ \\
\hline Abdomen & SMBG & MedARD & $8.0[4.0-16.2]$ & $8.1[4.5-14.2]$ & $8.3[4.0-16.3]$ \\
\hline Arm & SMBG & MedARD & $8.0[3.5-15.1]$ & $6.9[2.9-11.5]$ & $8.0[3.5-15.0]$ \\
\hline \multicolumn{3}{|c|}{ Number of CGM-YSI pairs } & 833 & 360 & 1330 \\
\hline \multicolumn{3}{|c|}{ Number of CGM-SMBG pairs } & 458 & 178 & 681 \\
\hline \multicolumn{3}{|c|}{$\begin{array}{l}\text { Mean }[95 \% \mathrm{CI}] \text { of } \mathrm{ARD}_{\mathrm{arm}} / \mathrm{ARD}_{\mathrm{abd}} \text { ratio for the } \\
\text { whole glycemic range }\end{array}$} & \multicolumn{2}{|c|}{$\begin{array}{c}\text { YSI reference } \\
0.80[0.44-1.75]\end{array}$} & $\begin{array}{l}\text { SMBG reference } \\
0.91[0.50-1.83]\end{array}$ \\
\hline
\end{tabular}

MARD is presented as mean ARD (SD) and MedARD as median ARD [interquartile range]. The CGM $\mathrm{abd}_{\text {and }} \mathrm{CGM}_{\text {arm }}$ do not have different MedARD (Skillings-Mack test, $P>0.05$ ).

ARD, absolute relative difference; CGM, continuous glucose monitor; SD, standard deviation; SMBG, self-monitoring of blood glucose; YSI, 2300 STAT, Yellow Springs Instrument. 


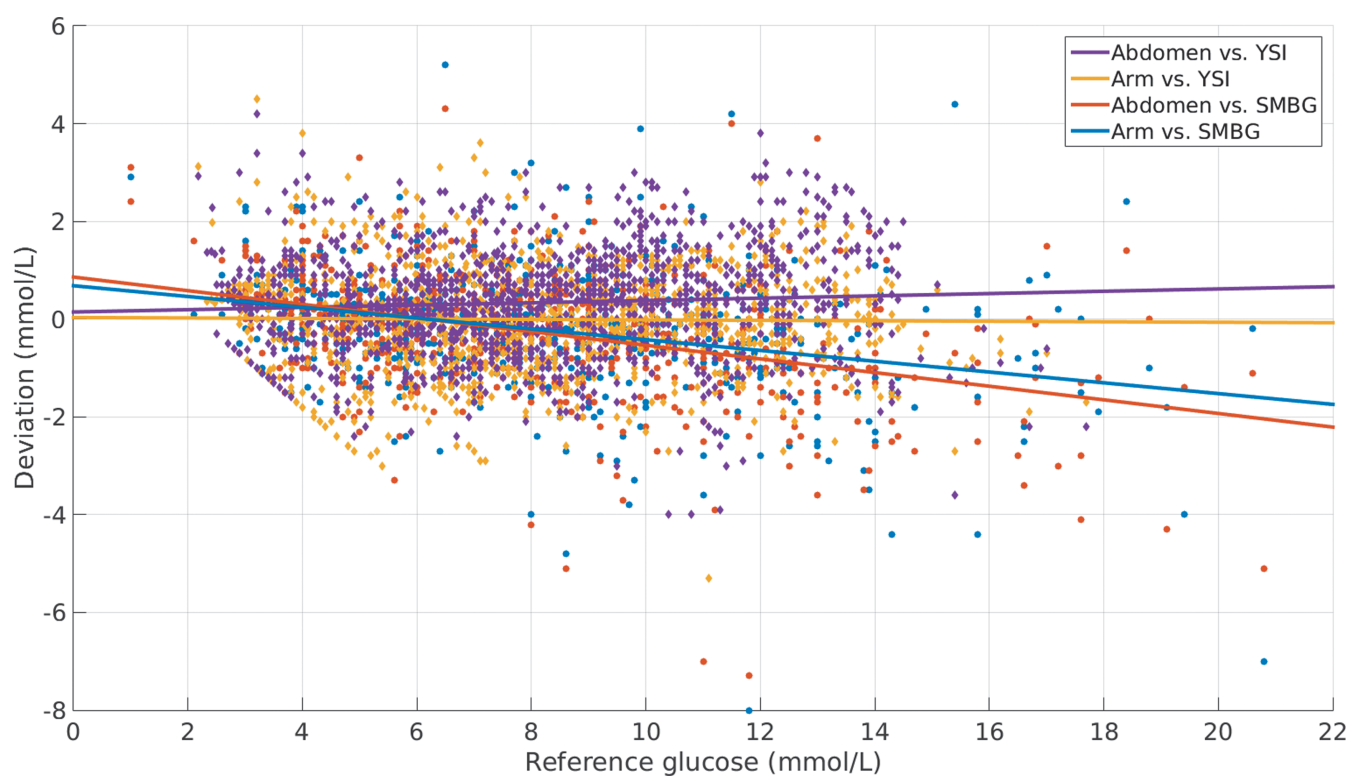

FIG. 1. Illustration of the deviation of CGM readings from the reference glucose plotted against the paired YSI and SMBG values. Scatter plot of the deviation=CGM glucose - reference glucose versus the reference glucose, and the least squares regression line fitted to the data. Each line is fitted to the data points with the same color. The data are from all patients and all visits. CGM, continuous glucose monitor; SMBG, self-monitoring of blood glucose; YSI, 2300 STAT, Yellow Springs Instrument.

\section{In-clinic visits}

Participants arrived at 8:00 AM after an overnight fast. An antecubital vein catheter was inserted and covered with a heating pad. Initial blood and urine samples were taken before the study start. Participants were served a meal containing 40 grams of carbohydrates, and they took a meal corrected insulin bolus. After $130 \mathrm{~min}$, participants were either cycling (two in-clinic visits) or resting (one in-clinic visit) for $45 \mathrm{~min}$ or until plasma glucose (PG) $\leq 3.9 \mathrm{mmol} / \mathrm{L}$ was reached. After the resting/cycling period the participants were observed for $125 \mathrm{~min}$. PG was analyzed on an YSI every $15 \mathrm{~min}$ for the first $130 \mathrm{~min}$, every $5 \mathrm{~min}$ during the cycling/resting period and the following $45 \mathrm{~min}$, and every $10 \mathrm{~min}$ for the last $60 \mathrm{~min}$ of the in-clinic visit.

\section{Follow-up period}

During 4 days after each in-clinic visit, participants were instructed to perform SMBG-measurements seven times a

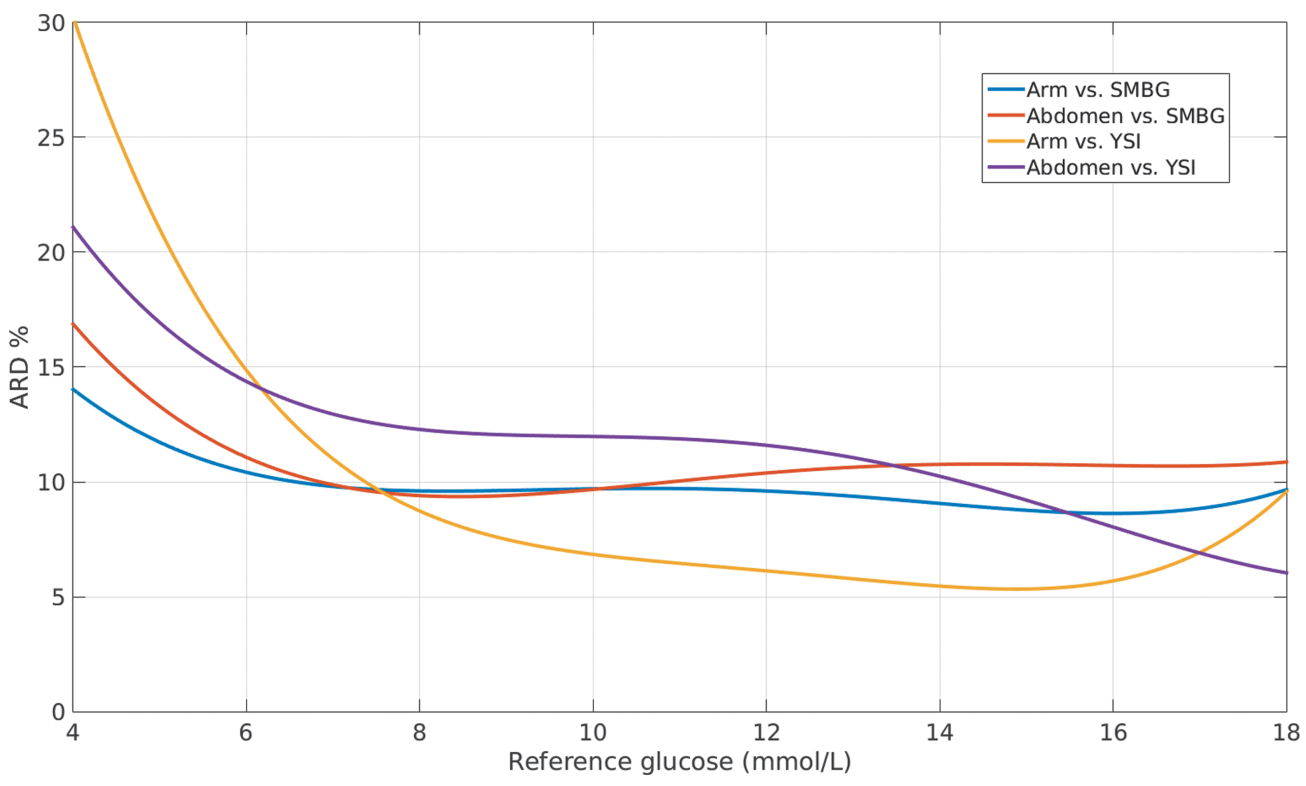

FIG. 2. The ARD of the CGM measurements from the reference comparator larger than $4 \mathrm{mmol} / \mathrm{L}$. The magnitude of the ARD depends on glucose level. The curves are fourth order polynomial fits to ARD. Using SMBG as comparator, the ARD of arm and abdomen are very similar. When YSI is the comparator the arm ARD is lower particularly in hyperglycemia as also indicated in Table 2. The data are from all patients and all visits. ARD, absolute relative difference. 
day on predefined time points (breakfast, $2 \mathrm{~h}$ after breakfast, lunch, $2 \mathrm{~h}$ after lunch, dinner, $2 \mathrm{~h}$ after dinner and 03:00 $\mathrm{AM}$ ) and to register the values. CGM and blood glucose BG meters were downloaded after the 4 days. All participants used the BG meter Contour Link or Contour Next (Bayer HealthCare LLC; Diabetes Care, Whippany, NJ), for SMBGs.

\section{Outcome accuracy metrics}

We used five accuracy metrics to assess and compare the performance of the $\mathrm{CGM}_{\mathrm{arm}}$ versus $\mathrm{CGM}_{\mathrm{abd}}$. The metrics were the absolute relative difference (ARD), precision absolute relative difference (PARD), Clarke error grid analysis (CEGA), sample-based sensitivity and specificity analyses, and event-based hypoglycemia detection. Outcome Accuracy Metrics section in Supplementary Data describes the detailed definitions and computations of the accuracy metrics.

\section{Data definitions}

The $\mathrm{CGM}_{\mathrm{arm}}$ and $\mathrm{CGM}_{\mathrm{abd}}$ measurements were paired with their time-matched YSI and SMBG measurements as reference. The matched pairs were determined by pairing the reference glucose value to its closest-in-time CGM reading. If the closest time was $>3 \mathrm{~min}$, the reference glucose was marked as unpaired and was not included in the study. Every reference value was paired with one $\mathrm{CGM}_{\mathrm{arm}}$ measurement and one $\mathrm{CGM}_{\mathrm{abd}}$ measurement, and therefore, the number of CGM-reference pairs was the same for arm and abdomen sensors. The CGM-SMBG pairs were from the CGM insertion time ( 2 days before in-clinic visits) until the end of data collection (4 days after the in-clinic visits). The SMBG measurements used for calibration were excluded from the analysis, because they are accurate by calibration definition. Hypoglycemia was defined as reference $\leq 3.9 \mathrm{mmol} / \mathrm{L}$, euglycemia was $3.9<$ reference $<10$, and hyperglycemia was reference $\geq 10 \mathrm{mmol} / \mathrm{L}$. Missing data for an in-clinic period and follow-up period were defined as (1) if either $\mathrm{CGM}_{\text {arm }}$ or $\mathrm{CGM}_{\mathrm{abd}}$ data were missing, (2) if a YSI measurement was missing, or (3) if an SMBG measurement was missing. Applying these 3 criteria remained 3 in-clinic visits with missing data for the comparison between the $\mathrm{CGM}_{\mathrm{arm}}$-YSI pairs and $\mathrm{CGM}_{\text {abd- }}$ YSI pairs, and it left 12 follow-up periods with missing data for the comparison between the $\mathrm{CGM}_{\mathrm{arm}^{-}}$ SMBG pairs and $\mathrm{CGM}_{\mathrm{abd}}-\mathrm{SMBG}$ pairs.

\section{Statistical analysis}

We used the Skillings-Mack two-factor $(2 \times 2)$ analysis of variance to investigate the difference between the accuracy of $\mathrm{CGM}_{\text {arm }}$ versus $\mathrm{CGM}_{\mathrm{abd} .}{ }^{6}$ The CGM insertion site (arm vs. abdomen) and the patients were the two factors, and the three treatment visits were the replications in the test. The SkillingsMack test was used due to the missing data in the block design and the non-normal distribution of the ARD, and sensitivity and specificity data (Lilliefors test for normality, $P<0.05$ ). This is a two-sided traditional comparative test with the null hypothesis that the accuracy of $\mathrm{CGM}_{\text {arm }}$ and $\mathrm{CGM}_{\mathrm{abd}}$ sensor measurements is identical. The main endpoint for the current study was the ARD comparison between $\mathrm{CGM}_{\mathrm{arm}}$ and $\mathrm{CGM}_{\mathrm{abd}}$ in the whole glycemic range. Other endpoints were the ARD comparison in hypoglycemia, hyperglycemia, and euglycemia, hypoglycemia sensitivity and specificity, and hyperglycemia
Table 3. Mean Absolute Difference and Median AbSOlute DifFERENCE FOR REFERENCE GLUCOSE $\leq 4.2 \mathrm{MMOL} / \mathrm{L}$

\begin{tabular}{lclc}
\hline Insertion site & Reference & Metric & \\
\hline Abdomen & YSI & MAD & $0.84(0.72)^{\mathrm{a}}$ \\
Arm & YSI & MAD & $0.89(0.72)$ \\
Abdomen & YSI & MedAD & $0.70[0.32-1.15]$ \\
Arm & YSI & MedAD & $0.70[0.40-1.20]$ \\
Abdomen & SMBG & MAD & $0.76(0.59)$ \\
Arm & SMBG & MAD & $0.76(0.67)$ \\
Abdomen & SMBG & MedAD & $0.6[0.3-1.1]$ \\
Arm & SMBG & MedAD & $0.6[0.3-0.95]$ \\
Number of CGM-YSI pairs & & 170 \\
Number of CGM-SMBG pairs & & 84
\end{tabular}

The $\mathrm{CGM}_{\mathrm{abd}}$ and $\mathrm{CGM}_{\mathrm{arm}}$ do not have different MedAD (Skillings-Mack test, $P>0.05$ ). MAD is presented as mean (SD) and MedAD as median [interquartile range].

${ }^{\mathrm{a}} \mathrm{mmol} / \mathrm{L}$.

MAD, mean absolute difference.

sensitivity and specificity. Statistical analyses were performed in MATLAB (version 8.5.0.197613 [R2015a]). To compare the $A R D$ of $\mathrm{CGM}_{\text {arm }}$ with ARD of $\mathrm{CGM}_{\mathrm{abd}}$, we calculated the ratio of $\mathrm{CGM}_{\text {arm }}$ ARDs to their paired $\mathrm{CGM}_{\mathrm{abd}}$ ARDs, that is, $\rho_{i}=\mathrm{ARD}_{\mathrm{arm}, i} / \mathrm{ARD}_{\mathrm{abd}, i}$ for $i=1, \ldots, n(n$ is the number of available ARD values) for the whole glycemic range, and we computed the empirical cumulative distribution function (CDF) of $\rho$ and its $95 \%$ confidence interval.

\section{Results}

Thirteen individuals performed three in-clinic visits and follow-up periods and one individual performed two in-clinic visits and a follow-up period. Table 1 presents the participants' demographic data.

The analysis included a total of 2660 CGM-YSI pairs (an average of $68 \mathrm{CGM}_{\mathrm{arm}}-\mathrm{YSI}+\mathrm{CGM}_{\mathrm{abd}}-$ YSI pairs per person per in-clinic visit) and a total of 1362 pairs of CGMSMBG (average of $45 \mathrm{CGM}_{\mathrm{abd}}-\mathrm{SMBG}+\mathrm{CGM}_{\mathrm{arm}}-\mathrm{SMBG}$ pairs per person per follow-up period). Table 2 presents the mean absolute relative difference (MARD) and median ARD of the $\mathrm{CGM}_{\text {abd }}$ and $\mathrm{CGM}_{\text {arm }}$ with respect to YSI and SMBG measurements and the number of CGM-reference pairs in different glycemic ranges. The total of in-clinic visits and followup periods across all the patients was 41 consisting of 3 in-clinic

\section{Table 4. Mean Absolute Relative Difference and Median Absolute Relative Difference During 45 MIN OF EXERCISE (CYCLING) FOR THE THIRD in-Clinic Visit (CyCling Followed BY GLUCAGON INJECTION)}

\begin{tabular}{lclc}
\hline Insertion site & Reference & Metric & \\
\hline Abdomen & YSI & MAD & $16.5(14.5)$ \\
Arm & YSI & MAD & $15.2(14.3)$ \\
Abdomen & YSI & MedAD & $11.9[7.1-22.5]$ \\
Arm & YSI & MedAD & $12.1[6.5-18.0]$ \\
\hline
\end{tabular}

The $\mathrm{CGM}_{\mathrm{abd}}$ and $\mathrm{CGM}_{\text {arm }}$ do not have different MedAD (Skillings-Mack test, $P>0.05$ ). MARD is presented as MARD (SD) and MedARD as median ARD [interquartile range]. 
Table 5. Clark Error Grid Analysis for the Whole Glycemic Range

\begin{tabular}{|c|c|c|c|c|c|c|c|c|c|c|c|c|c|c|c|c|c|}
\hline \multirow[b]{2}{*}{ Insertion site } & \multirow[b]{2}{*}{ Reference } & \multicolumn{3}{|c|}{ Hypoglycemia } & \multicolumn{3}{|c|}{ Euglycemia } & \multicolumn{5}{|c|}{ Hyperglycemia } & \multicolumn{5}{|c|}{ Whole range } \\
\hline & & $A$ & $D$ & $E$ & $A$ & $B$ & $C$ & $A$ & $B$ & $C$ & $D$ & E & $A$ & $B$ & $C$ & $D$ & $E$ \\
\hline Abdomen & YSI & 73.0 & 27.0 & 0 & 86.1 & 11.6 & 2.3 & 91.4 & 8.6 & 0 & 0 & 0 & 85.6 & 11.6 & 0 & 2.8 & 0 \\
\hline Arm & YSI & 76.6 & 23.4 & 0 & 82.1 & 17.9 & 0 & 98.0 & 2.0 & 0 & 0 & 0 & 86.0 & 11.6 & 0 & 2.4 & 0 \\
\hline Abdomen & SMBG & 77.8 & 22.2 & 0 & 83.0 & 17.0 & 0 & 88.8 & 11.2 & 0 & 0 & 0 & 84.1 & 14.4 & 0 & 1.5 & 0 \\
\hline Arm & SMBG & 75.6 & 24.4 & 0 & 83.8 & 16.2 & 0 & 90.4 & 8.4 & 0 & 0.6 & 0.6 & 85.0 & 13.1 & 0 & 1.8 & 0.1 \\
\hline
\end{tabular}

The values are percentage of the points in the zones.

visits for each of the 13 patients and 1 patient with 2 visits. The Skillings-Mack test did not indicate any statistically significant difference between $\mathrm{CGM}_{\mathrm{abd}}$ and $\mathrm{CGM}_{\mathrm{arm}}$ in any of the tested data $(P>0.05)$. The overall median ARD of the sensor inserted on arm tended to be lower than on the abdomen without being statistically different (Table 2). Using the CDF, we computed the mean and $95 \%$ confidence interval of this ratio which are presented in the last two rows of Table 2 . The results show that although the mean of this ratio is smaller than one, which implies the lower ARD of $\mathrm{CGM}_{\text {arm }}$ compared to $\mathrm{CGM}_{\mathrm{abd}}$, its confidence interval includes values larger than one, which indicates that we cannot conclude on the lower ARD of $\mathrm{CGM}_{\text {arm }}$.

The least squares regression line in Figure 1 and Supplementary Fig. S1 indicate that the CGM readings slightly overestimate SMBG values in hypoglycemia and underestimate the SMBG values in hyperglycemia. In Figure 2 and Supplementary Fig. S2, when YSI is the comparator, the arm ARD is lower, particularly in hyperglycemia. When SMBG is the comparator, the ARD of arm and abdomen are very similar. Figure 3 and Supplementary Fig. S3 show the contour plot of the bivariate probability distribution of arm and abdomen CGM measurements. There is no statistical difference between the median ARD (MedARD) of $\mathrm{CGM}_{\mathrm{arm}}$ and $\mathrm{CGM}_{\text {abd }}$ (Table 3). The mean absolute difference (MAD) and MedARD during exercise (Table 4) are higher than the general ARD and MedARD in Table 2. This suggests a po- tential drop in CGM accuracy due to exercise. However, even during exercise, there is no difference between arm and abdomen ARD (Skillings-Mack test, $P>0.05$ ).

Supplementary Fig. S4A-D shows the plots of CEGA for the whole glycemic range. The percentage of the CGM readings in CEGA zone A for the arm sensor was slightly higher than that for the abdomen sensor (Table 5). The mean (SD) of the PARD between the two CGM sensors was $10.6 \% \pm 11.7 \%$. Figure 3 illustrates the contour plots of the bivariate Gaussian distribution of abdomen CGM versus arm CGM measurements along with the $95 \%$ confidence region of the distribution. The plot is an indicator of the precision of the sensors. A wider confidence region indicates lower precision and vice versa. Using YSI as reference glucose, the hypoglycemia sensitivities of arm and abdomen sensors were similar, while the hyperglycemia sensitivity of arm sensor was higher (Table 6 and Fig. 4). Furthermore, using SMBG as reference glucose, the hypoglycemia sensitivity of the arm sensor was slightly higher than the abdomen sensor. Nevertheless, none of these differences was statistically significant. Supplementary Fig. S5A and B illustrates an example of the YSI and SMBG event-based hypoglycemia detection by $\mathrm{CGM}_{\mathrm{abd}}$ and $\mathrm{CGM}_{\mathrm{arm}}$ sensors. The $\mathrm{CGM}_{\mathrm{arm}}$ and $\mathrm{CGM}_{\mathrm{abd}}$ sensors can detect 10 YSI hypoglycemic events out of 27 hypoglycemic events (Table 7). The $\mathrm{CGM}_{\text {arm }}$ sensor detects one more SMBG hypoglycemic event than the $\mathrm{CGM}_{\mathrm{abd}}$ sensor.

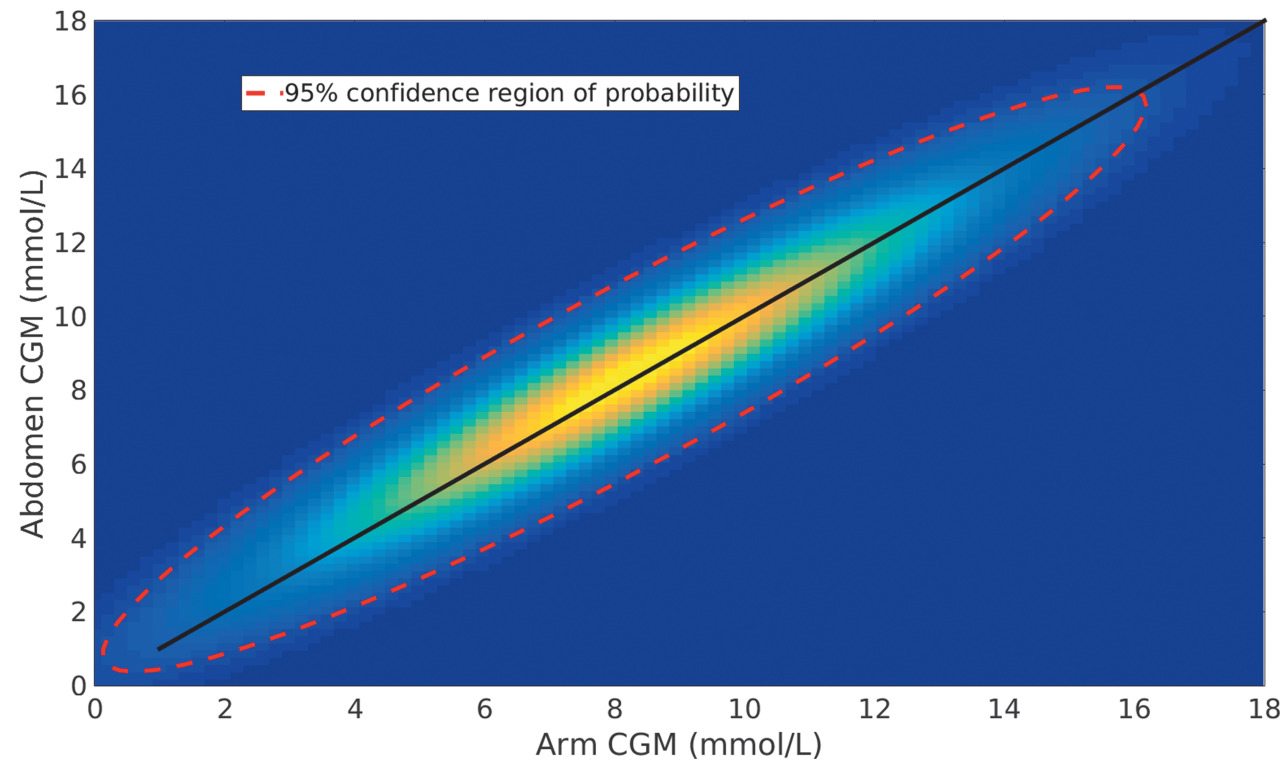

FIG. 3. The contour plot of the bivariate probability distribution of arm and abdomen CGM measurements. 


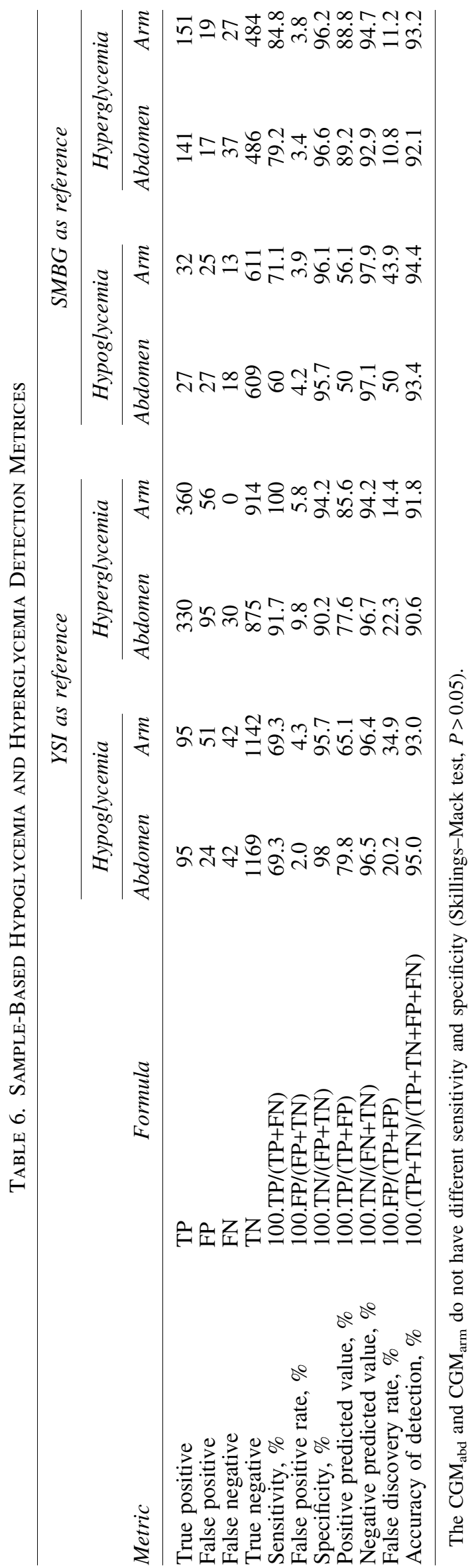

\section{Discussion}

We compared the accuracy of the Dexcom G4 Platinum CGM sensor inserted on the upper arm (off-label) with the G4 CGM sensor inserted on the abdomen (approved sensor site) in adults with type 1 diabetes. YSI and SMBG measurements were used as reference for blood glucose concentrations. We found no significant difference in accuracy between $\mathrm{CGM}_{\mathrm{arm}}$ and $\mathrm{CGM}_{\text {abd }}$. Although not statistically significant, the arm sensor tended to outperform the abdomen sensor for almost all studied accuracy metrics.

We used YSI as an additional reference measure for the comparisons, where the only study to our knowledge studying accuracy at the upper arm used SMBG as reference value. ${ }^{6}$ The YSI device is a laboratory analyzer using GOD Enzyme for reference glucose concentration. SMBG meters have a lower accuracy and lower precision than YSI analyzers; however, they reflect the free-living condition. We included both YSI and SMBG in this study. The error of the SMBG meters is different depending on the manufacturer. Therefore, when comparing the results of the CGM accuracy studies, the error associated with the SMBG meters from different manufacturers should be considered. For this study, we used the Contour Link or Contour Next, which was shown to have the highest accuracy among all BG meters. ${ }^{7}$

The CGM sensors measure the interstitial concentration of glucose. There are no reasons to believe that there should be a difference between interstitial tissues on the upper arm and abdomen. However, some sensors are only approved to one or the other site. Furthermore, there may be a difference in the concentrations of glucose in the interstitial tissues during nonsteady state glucose levels, for example, due to difference in local blood flow during exercise or difference in risk for minor mechanical displacements. It was exactly what we demonstrated by finding a higher MAD and MedARD during exercise; however, we found no statistically significant difference in accuracy between the two sensor sites during exercise. We have seen individuals with type 1 diabetes in our outpatient clinic who use their CGM sensor on the arm for Dexcom G4, even though it is not approved for this insertion site. Based on the results presented here, there is no reason to discourage this use.

Several other studies are available regarding accuracy determination in newer generation CGMs. As mentioned before, Faccioli et al. ${ }^{4}$ compared the accuracy of Dexcom G5 CGM sensor in abdomen, gluteus, and arm with respect to SMBG measurements. Their study used the old G4 algorithm not the current G4/505 algorithm. The old G4 algorithm is less accurate than the current G4/G5 software. The results did not detect any accuracy difference (the MARD was $13.3 \%$, $13.4 \%$, and $12.9 \%$ for abdomen, arm, and gluteus, respectively), which is aligned with our results indicating no difference in the accuracy of arm sensor versus abdomen sensor in adults or when using SMBG or YSI as comparator. Boscari et al. ${ }^{8}$ compared the general accuracy of Dexcom G4 Platinum with FreeStyle Libre, and the G4 Platinum sensor had the old G4 algorithm. Using SMBG as reference, the overall MARD of Dexcom G4 Platinum was $12.9 \%$. Like our results, they also reported a relatively large ARD in hypoglycemia for the sensor. Their results indicated a MARD of $24.0 \%$, $12.0 \%$, and $10.8 \%$, in hypoglycemia, euglycemia, and hyperglycemia, respectively. In our study, for the CGM placed 
Hypoglycemia sensitivity

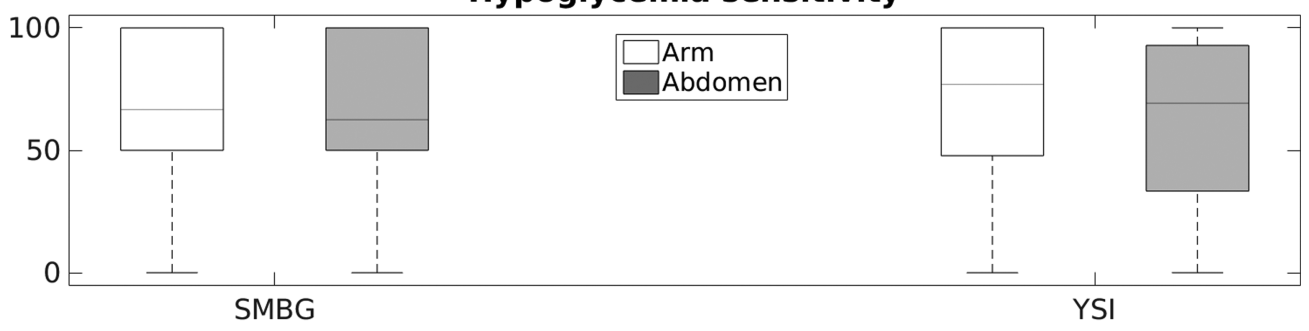

Hyperglycemia sensitivity

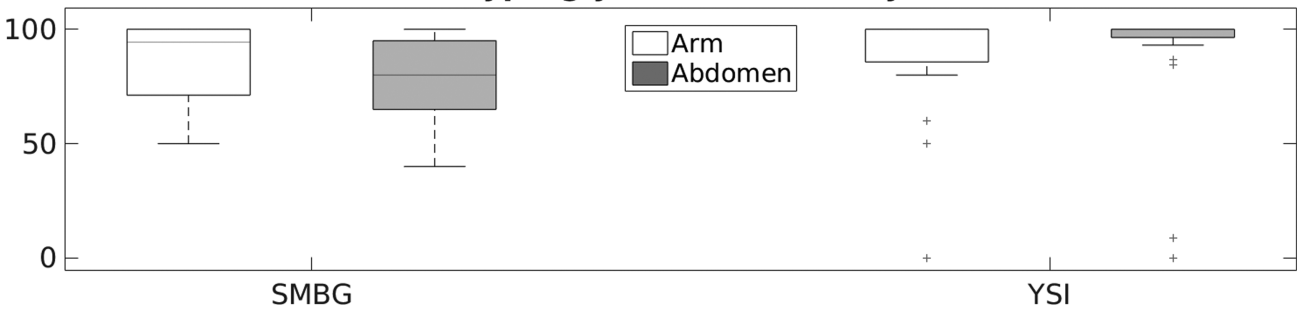

FIG. 4. Boxplot of hypoglycemia (reference glucose $\leq 3.9 \mathrm{mmol} / \mathrm{L}$ ) sensitivity and hyperglycemia (reference glucose $\geq 10 \mathrm{mmol} / \mathrm{L}$ ) sensitivity with SMBG and YSI as comparators. The data are from all patients and all visits.

on abdomen, the SMBG-based MARD in the overall range, hypoglycemia, euglycemia, and hyperglycemia was $12.5 \%$, $32.9 \%, 11.4 \%$, and $10.3 \%$, respectively. Taleb et al. ${ }^{9}$ report an overall mean/median ARD of $13.77 \% / 13.34 \%$ for Dexcom G4 Platinum with respect to YSI measurements. In the study by Aberer et al., ${ }^{10}$ the overall MARD of Dexcom G4 Platinum system was $16.8 \%$ with respect to venous PG readings, and the MARD in hypoglycemia, euglycemia, and hyperglycemia was $23.8 \%, 16.3 \%$, and $11.6 \%$, respectively. Nakamura and Balo ${ }^{11}$ investigated the accuracy of Dexcom G4 Platinum sensor with respect to YSI and SMBG measurements. According to their results, using YSI as reference, the overall MARD was $13 \%$ and the median ARD was $10 \%$, and the precision ARD between the two sensors-both placed on the approved site-was 9\%. Their reported hypoglycemia sensitivity of $83 \%$ and the hyperglycemia sensitivity of $97 \%$ are larger than the corresponding results in our study. Furthermore, the percentage of the CGM readings in zone A of CEGA was $81 \%$, which is comparable with the $85.6 \%$ achieved in our study $\left(\mathrm{CGM}_{\mathrm{abd}}\right.$ with YSI as reference). Matuleviciene et al. ${ }^{12}$ investigated the accuracy of G4 Platinum in comparison with the Enlite sensor in the Medtronic Guardian REAL-Time CGM system. With respect to SMBG, the overall MARD was $13.9 \%$ and the MARD in hypoglycemia was $20.0 \%$. Having a larger MARD in hypoglycemia compared to the rest of the glycemic range is a known fact, and several CGM accuracy studies indicated a

Table 7. Hypoglycemic Event Detection

\begin{tabular}{|c|c|c|c|c|c|}
\hline \multirow[b]{2}{*}{ Reference } & \multirow{2}{*}{$\begin{array}{c}\text { Number } \\
\text { of } \\
\text { hypoglycemic } \\
\text { events }\end{array}$} & \multicolumn{2}{|c|}{$\begin{array}{c}\text { True } \\
\text { positive }\end{array}$} & \multicolumn{2}{|c|}{$\begin{array}{c}\text { False } \\
\text { negative }\end{array}$} \\
\hline & & Abdomen & Arm & Abdomen & Arm \\
\hline YSI & 27 & 10 & 10 & 17 & 17 \\
\hline SMBG & 45 & 30 & 31 & 15 & 14 \\
\hline
\end{tabular}

large MARD in hypoglycemia for the enzyme-based CGM systems. $^{10,13-15}$ In the study by Acciaroli et al., ${ }^{16}$ the G4 Platinum sensor had a MARD of $11.59 \%$ with respect to YSI measurements. They applied a Bayesian calibration approach which reduced the MARD to $8.5 \%$ for one-per-day calibration scenario. Our analysis indicates a PARD of $10.6 \%$ between $\mathrm{CGM}_{\text {arm }}$ and $\mathrm{CGM}_{\text {abd }}$ using G4 sensors. The study by Pleus et al. ${ }^{17}$ suggests a PARD of $7.3 \%$ between the two Dexcom G4 sensors both placed on abdomen. It seems as if the PARD between two sites is higher than for the same location comparisons, however, our other comparisons do not indicate that the sensor location affects sensor precision. In our study, the MedARD was $66 \%-76 \%$ of the MARD for CGM readings, supporting the findings from previous studies. ${ }^{18}$

One of the limitations of this work is that it included only 14 patients. Therefore, the study may be slightly underpowered for definitive conclusions. Although each patient had a high number of measurements included in the analysis, we need a larger subject population to be more conclusive about the $\mathrm{CGM}_{\text {arm }}$ accuracy in comparison with the $\mathrm{CGM}_{\mathrm{abd}}$ accuracy. We have not evaluated whether participants preferred to use the CGM on the arm for the abdomen. Finally, it should be notified that we started the analyses on sensor wear day 3 and thereby did not analyze the accuracy in the first 2 days of sensor use.

\section{Conclusions}

The abdomen is the only approved insertion site for the Dexcom G4 Platinum sensor. However, many patients insert the sensor on the arm. We investigated in adults whether the accuracy of the G4 continuous glucose monitoring sensor inserted on the arm is different from the accuracy of the G4 sensor inserted on the abdomen. We found the same accuracy at the two insertion sites whether using SMBG or YSI for comparison. 


\section{Acknowledgments}

This study was funded by the Danish Diabetes Academy funded by the Novo Nordisk Foundation. The authors thank the study participants and acknowledge the laboratory assistance by Alis Sloth Andersen (Department of Endocrinology Research, Copenhagen University Hospital Hvidovre, Denmark) and Jakob Bjerager and Jens Laigard (medical students, Copenhagen University).

\section{Author Disclosure Statement}

None of the investigators has personal or financial interests in the conduct or the outcomes of the project. I.I.K.S. has received speaker grants from Roche Diabetes Care and Rubin Medical. Z.M., A.R., and J.B.J. have no conflict of interest to declare. S.S. has served on the continuous glucose monitoring advisory board of Roche Diabetes Care. K.N. serves as adviser to Medtronic, Abbott and Novo Nordisk, owns shares in Novo Nordisk, has received research grants from Novo Nordisk and Roche Diabetes Care and has received fees for speaking from Medtronic, Roche Diabetes Care, Rubin Medical, Sanofi, Novo Nordisk, Zealand Pharma, and Bayer.

I.I.K.S., Z.M., A.R., S.S., and K.N. conceived the idea and designed the study. I.I.K.S. performed the studies, analyzed the data, and wrote and edited the article. Z.M. analyzed the data wrote and edited the article. A.R. performed some of the studies, analyzed the data, and reviewed and edited the article. S.S., K.N., and J.B.J. reviewed, edited, and approved the final article. I.I.K.S., Z.M., and K.N. take full responsibility for the content of this article.

\section{Supplementary Material}

Supplementary Data

Supplementary Figure S1

Supplementary Figure S2

Supplementary Figure S3

Supplementary Figure S4

Supplementary Figure S5

\section{References}

1. Slattery D, Choudhary P. Clinical use of continuous glucose monitoring in adults with type 1 diabetes. Diabetes Technol Ther 2017 May; 19(S2):S55-S61.

2. Beck RW, Riddlesworth T, Ruedy K, et al.: Effect of continuous glucose monitoring on glycemic control in adults with type 1 diabetes using insulin injections. JAMA 2017;317:371-378.

3. Danne $\mathrm{T}$, Nimri R, Battelino $\mathrm{T}$, et al.: International consensus on use of continuous glucose monitoring. Diabetes Care 2017;40:1631-1640.

4. Faccioli S, Del Favero S, Visentin R, et al.: Accuracy of a CGM sensor in pediatric subjects with type 1 diabetes. Comparison of three insertion sites: arm, abdomen, and gluteus. J Diabetes Sci Technol 2017;11:1147-1154.

5. Steineck IIK, Ranjan A, Schmidt S, et al.: Preserved glucose response to low-dose glucagon after exercise in insulinpump-treated individuals with type 1 diabetes: a randomised crossover study. Diabetologia 2019;62:582-592.
6. Chatfield M, Mander A: Europe PMC Funders Group the Skillings-Mack test (Friedman test when there are missing data). Stata J 2009;9:299-305.

7. Ekhlaspour L, Mondesir D, Lautsch N, et al.: Comparative accuracy of 17 point-of-care glucose meters. J Diabetes Sci Technol 2017;11:558-566.

8. Boscari F, Galasso S, Facchinetti A, et al.: FreeStyle Libre and Dexcom G4 Platinum sensors: accuracy comparisons during two weeks of home use and use during experimentally induced glucose excursions. Nutr Metab Cardiovasc Dis 2017;28:180-186.

9. Taleb N, Emami A, Suppere C, et al.: Comparison of two continuous glucose monitoring systems, Dexcom G4 Platinum and Medtronic Paradigm Veo Enlite System, at rest and during exercise. Diabetes Technol Ther 2016;18:561-567.

10. Aberer F, Hajnsek M, Rumpler M, et al.: Evaluation of subcutaneous glucose monitoring systems under routine environmental conditions in patients with type 1 diabetes. Diabetes Obes Metab 2017;19:1051-1055.

11. Nakamura K, Balo A: The accuracy and efficacy of the Dexcom G4 Platinum continuous glucose monitoring system. J Diabetes Sci Technol 2015;9:1021-1026.

12. Matuleviciene V, Joseph JI, Andelin M, et al:: A clinical trial of the accuracy and treatment experience of the Dexcom G4 Sensor (Dexcom G4 System) and Enlite Sensor (Guardian REAL-Time System) tested simultaneously in ambulatory patients with type 1 diabetes. Diabetes Technol Ther 2014;16:759-767.

13. Laffel L: Improved accuracy of continuous glucose monitoring systems in pediatric patients with diabetes mellitus: results from two studies. Diabetes Technol Ther 2016;18: 223-233.

14. Mahmoudi Z, Dencker Johansen M, Christiansen JS, et al.: A Multistep algorithm for processing and calibration of microdialysis continuous glucose monitoring data. Diabetes Technol Ther 2013;15:825-835.

15. Mahmoudi Z, Jensen MH, Dencker Johansen M, et al.: Accuracy evaluation of a new real-time continuous glucose monitoring algorithm in hypoglycemia. Diabetes Technol Ther 2014;16:667-678.

16. Acciaroli G, Vettoretti M, Facchinetti A, et al.: Toward calibration-free continuous glucose monitoring sensors: Bayesian calibration approach applied to next-generation dexcom technology. Diabetes Technol Ther 2018;20:59-67.

17. Pleus S, Schmid C, Link M, et al.: Performance evaluation of a continuous glucose monitoring system under conditions similar to daily life. J Diabetes Sci Technol 2013;7: 833-841.

18. Rodbard D: Characterizing accuracy and precision of glucose sensors and meters. J Diabetes Sci Technol 2014;8: 980-985.

Address correspondence to: Isabelle Isa Kristin Steineck, MD Steno Diabetes Center Copenhagen Niels Steensensvej 2-4 2820 Gentofte Denmark

E-mail: isabelle.isa.kristin.steineck@regionh.dk 